\title{
Altering targeted segmented past memory hierarchy with cognitive neuropsychology cognition treatment is now initiating a spontaneous remission in mental disorders.
}

\author{
Matthew David Dovel* \\ Division of International Suicide Prevention, Department of Psychology, USA
}

Accepted on September 19, 2017

All concepts covered in this article are experientially based on thirty years of research, development and application. Attempts have been made to purposefully to avoid esoteric language when describing the pathology of the mind for a wider audience [1].

"The day science begins to study non-physical phenomena; it will make more progress in one decade than in all the previous centuries of its existence." — Nikola Tesla

It is estimated that around (1) million people commit suicide and over (300) million report being affected by depression each year $[2,3]$.

My CV contains over 30 years of computer science training that ironically models some new conceptual abstract brain functionality theories [4]. This prospective allows me to better understand pathway and flow chart abstracts in memory development, storage, recall and modification. As a 'hacker', I often utilized a technique called 'reverse engineering' to have unauthorized access to programs and complex systems and it also has been useful for understanding the functions of the mind, brain and emotional connection $[5,6]$.

In my early studies I was frustrated with traditional psychology as it was not achieving the desired results I had hoped it would. Therefore, I started doing my own research in behavioral modification in the late 80 's by studying radical belief altering techniques: military experiments and training, Roman social conformity methodology, the German Nazi's and compliance techniques, and unethical behavioral modification studies. Then I totally went off the map by studying disciplines that created behavioral changes very quickly: hypnosis, [NLP] Neuro-linguistic programming, Sleight of mouth, religions, witchcraft, magic, [ESP] Extra Sensory Perception, cognitive bias tendencies, Voodoo, pickpocketing, healers, shaman and others. All of these disciplines, I have found, had a common link.

I learned that humans all have a fail-safe program built into our mind concerning where and how memories get stored. I had discovered a pattern. I identify very closely with Nikola Tesla in his obsession with the numbers $(3,6,9)$ and 'vortex math' [7]. The mind is mathematical and mechanical, but it is also very complex and abstract, requiring multiple views/models to demonstrate functionality of just one aspect.

We know that the frontal cortex is extremely important, and it serves many functions, but it may surprise you to know that memory storage is not one of them. The frontal cortex serves as an access point to our past memories. And, these access points also have links to a unique emotional response in the limbic system, with each memory. As long as we are in a cognitive conscious state memories are being formed, and streamed into the subconscious all being stored in one of two categories. Regardless of the intensity of a past experience an emotional state gets attached to all memories, even if we are apathetic at the time of the experience so, even apathy is assigned to a category.

The hierarchical value and meaning of memories are determined during their formation by the length of time they're held in the conscious mind. The longer an experience is being viewed in the now, as a new experience without reference, 15 min max (any experience of exceeding $15 \mathrm{~min}$ risks psychosis), the greater the hierarchical value a memory will have. Any experience above a level 8 , on a scale of between 1 to 10 and a 10 being the highest level is considered to be a 'memory trauma' [8]. And, any memory above a level 4 does not get fully processed into the subconscious mind [unless the memory code is applied purposefully or by accident]. $5 \%$ of the population has evolved to utilized memory coding or, had figured it out at an early age in their development to use it as a coping skill.

For the purpose of this discussion, hierarchical values are related mostly to the intensity of a past memory, and not the facts therein.

A 'memory trauma' above level 8 will develop over time into one of two directions: pleasure seeking, or pain avoidance. Pain avoidance for past trauma creates some of the following symptoms: depression, anxiety, phobias, manic, bipolar, P.T.S.D. and suicidal ideation. These types of mental disorders always have an inception point, identifying them as a learned response.

It is now possible with cognitive neuropsychology treatments [9] (Nu-Rekall ${ }^{\circledR}$ mind science technologies), to quickly alter past targeted memory hierarchy utilizing a newly discovered cognition mathematical coding. This cognition coding changes the intensity of targeted memories by changing the old 'emotion and intensity' attached state, or association, to an experience with a new emotional association. The process takes less than $5 \mathrm{~min}$, initiating a spontaneous remission in the mental disorder being targeted. These new associations are permanent.

As the mind, brain and emotions are conjoined in a mathematical formula we see an untenable treatment that can be eventually 
Citation: Dovel MD. Altering targeted segmented past memory hierarchy with cognitive neuropsychology cognition treatment is now initiating a spontaneous remission in mental disorders. J Clin Psychiatry Cog Psychol. 2017;1(1):1-2.

facilitated easily in one of three ways; by mental health professionals, self-help and covertly. As $85 \%$ of all mental disorders are symptomatic of past memory trauma, it is now foreseeable that in the next decade we could see a drastic reduction unwanted mental disorders.

We are currently at a (2) trillion dollar cost for treating mental disorders globally each year. Most of these resources will soon be forecast to be allocated in the needs of other positive human development [10].

Of course, even though these Nu-Rekall ${ }^{\circledR}$ mind science technology treatments were developed, tested and implemented in 2005, they're still successfully treating mental disorders today. Nu-Rekall ${ }^{\circledR}$ mind science technologies has published and unpublished studies, since 2005 for proprietary source documentation purposes and, it is now time to move into clinical study testing for applied evidence based approval.

$\mathrm{Nu}-\mathrm{Rekall}^{\circledR}$ mind science technologies contain published and unpublished works that are copyrighted and trademarked, is a complete unique system to address mental disorders that is nonmedical and noninvasive.

\section{References}

1. Quote by Nikola Tesla, Accessed from: https://www. goodreads.com/quotes/139502-the-day-science-begins-tostudy-non-physical-phenomena-it-will

2. Nordqvist C (2011) One million people commit suicide each year. Medical News Today, Accessed on 10th September 2011
3. World Health Organization (2017) Mental disorders. Accessed from: http://www.who.int/mediacentre/ factsheets/fs396/en/ World Health Organization. Accessed on : April 2017.

4. https://www.linkedin.com/in/suicidepreventionexpert/

5. National Institute of Medical Health (2015) Mental Health Awareness Month: Trillion Dollars spent on mental health, Accessed from: https:/www.nimh.nih.gov/about/directors/ thomas-insel/blog/2015/mental-health-awareness-monthby-the-numbers.shtml National Institute of Medical Health. Accessed on May 15, 2015.

6. International Suicide Prevention (2014) Self-help 4 phase. International Suicide Prevention. Accessed from: http:// www.supportisp.org/4phase.html. Accessed on September $10,2014$.

7. https://en.wikipedia.org/wiki/Traumatic_memories Wikipedia, the free encyclopedia. Accessed on May 27, 2017

8. International Suicide Prevention a 501(c)(3) public charity: http://www.supportisp.org

9. Nu-Rekall ${ }^{\circledR}:$ http://www.nu-rekall.com

10. Insel T (2015) Post by Former NIMH Director Thomas Insel: Mental Health Awareness Month: By the Numbers. from NIH National Institute of Mental Health: https://www. nimh.nih.gov/about/directors/thomas-insel/blog/2015/ mental-health-awareness-month-by-the-numbers.shtml Accessed on August 3, 2016.

\section{*Correspondence to:}

Matthew David Dovel

Division of International Suicide Prevention

Department of Psychology

USA

E-mail: president@supportisp.org 